\title{
Synthesizing Graphene Oxide as a Working Electrode Using Cyclic Voltammetry
}

\author{
Aldwin Amireza, Pirim Setiarso* \\ Chemistry Department, Faculty of Mathematics and Natural Science \\ Universitas Negeri Surabaya \\ Surabaya, Indonesia \\ pirimsetiarso@unesa.ac.id
}

\begin{abstract}
This research has been done by synthesizing graphene oxide as a working electrode using Hummer method. Aim of this study is comparing capability of electron transfer within electrode. This can be done with using composition of working electrode by mixing synthesized product and organic glue paraffin, stands for 8:2. The comparison has been used to knowing the optimum condition of working electrode. Continued to the next test using Cyclic Voltammetry (CV), determined by deposition time in $10 \mathrm{~s}$ and scan rate in 300 $\mathrm{mV} / \mathrm{s}$. Sample solution in condition test is using alkaline solution in $\mathbf{p H}$ 9. This has purpose to compare working electrode carbon paste in common between better working electrode using graphene oxide. The result is proved with counting coefficient diffusion at optimum voltamogram condition and indicated graphene oxide electrode has working effectivity better than working electrode that contained carbon paste.
\end{abstract}

Keywords-- graphene oxide, coefficient diffusion, cyclic voltammetry, carbon paste electrode

\section{INTRODUCTION}

Graphene oxide (GO) is a new form of synthesized graphite, which is an oxidized multilayer because chemical reaction that involved using oxidizing agent, potassium permanganate [1]. Differences between graphite and graphene oxide shown by structural layers that formed, thereby graphite is a hexagonal monolayer carbon using $\mathrm{sp}^{2}$ hybridization. Graphene is defined by the International Union for Pure and Applied Chemistry (IUPAC) as "a single carbon layer of graphite structure, describing its nature by analogy to a polycyclic aromatic hydrocarbon of quasi-infinite size". For example, electrons interact with atomic carbon. Graphite is a 3D carbon based material made up of millions of layers of graphene. Oxidation of graphite has been used strong oxidizing agents, oxygenated functionalities are introduced into the graphite structure which not only expands the layer separation, but also makes the material hydrophilic. This property enables the graphite oxide to be exfoliated in water using sonication, ultimately producing single or few layer graphene, known as graphene oxide (GO). The main difference between graphite oxide and GO is, thus, the number of layers. In GO dispersion, a few layer flakes and monolayer flakes can be found.

One of the advantages of GO is the ease of dispersability in water and other organic solvents, as well as in different matrixes. This is due to the presence of the oxygen functionality groups. This remains a very important property in mixing the material with ceramic or polymer matrixes when trying to improve their electrical and mechanical properties [2] [3]. Meanwhile, in terms of electrical conductivity, GO is often described as an electrical insulator, due to the disruption of its $\mathrm{sp}^{2}$ bonding networks.

Voltammetry has some advantage from other method. This examined advantage for voltammetry is not really necessary to do some long sample preparation, like splitting between major and minor elements and concentration before analyzing [4]. A solid electrode has a wide potential anode and cathode range and often used is carbon paste electrode. Carbon paste electrode is a cheap electrode and easy to renew and produced in a small shape. To enhance the working of carbon paste electrode thus do modification with material that advance of accomplished for analyzing some metal. Modifier that used to enhance selectivity of carbon paste electrode is a complex compound [5]. Cyclic voltammetry (CV) has become an important and widely used electroanalytical technique in many areas of chemistry. It is rarely used for quantitative determinations, but it is widely used for the study of redox processes, for understanding reaction intermediates, and for obtaining stability of reaction products. [6] GO synthesis result has been used as a working electrode on alkaline water electrolysis's circuit.

\section{MATERIALS AND PROCEDURES}

\section{A. Equipment and Materials}

Material that used in this research is graphite powder 300 mesh, sulfuric acid 98\%, phosphoric acid 65\%, solid 
potassium permanganate, hydrogen peroxide $30 \%$, chloric acid $37 \%$ and $1 \mathrm{M}$, zinc powder, aquademineral, aquadest, ethanol p.a. Equipment that used are glassware, electrolyzing circuit, $\mathrm{pH}$ meter, oven, set of Voltammetry Instrument VA737, XRD Phillips PANalytical, FTIR, SEMEDX JEOL JSM 6510 LA. Instrumental test has been done at The State University of Surabaya, Institute Technology of Sepuluh November Surabaya, and National Nuclear Power Agency (BATAN).

\section{B. Synthesizing process of graphene oxide}

First thing to do in synthesizing GO is mixing $1 \mathrm{~g}$ graphite powder with sulfuric acid and phosphoric acid in 9:1 ratio on $100 \mathrm{~mL}$ total volume. The mixture continuing to be stirred using hot plate stirrer in medium speed without temperature for 6 hours. After that, the mixture that still in stirring condition then added by 6 grams of $\mathrm{KMnO}_{4}$ and stir for 18 hours by changing the mixture turned into condense brown mixture. The mixture then added by $1 \mathrm{~mL} \mathrm{H}_{2} \mathrm{O}_{2}$ in droplets, along stirring process. All the mixture finally filled with $400 \mathrm{~mL}$ aqua $\mathrm{DM}$, and the mixture changed into purple brown. Then, the mixture deposited a few days until the residue can be used as a graphite oxide source by decantation process. Residue then washed using centrifuge in $4000 \mathrm{rpm}$ speed. The washer for residue in centrifuge process is aqua DM, $\mathrm{HCl} 1 \mathrm{M}$ and ethanol p.a. After the residue has been washed and heated using oven in temperature $70^{\circ} \mathrm{C}$ for 24 hours.

Graphite oxide then mixed with scaling $50 \mathrm{mg}$ over 50 $\mathrm{mL}$ aqua $\mathrm{DM}$ in ultrasonic bath for 2 hours. Sonication stopped and then added $10 \mathrm{~mL}$ solution of $\mathrm{HCl} \mathrm{37 \% ,0.8 \textrm {g }}$ of zinc powder and $10 \mathrm{~mL}$ solution again with stirring time 30 minutes for each addition, gradually. While stirring in constant speed using hot plate stirrer, the mixture would be formed as a thin layer called graphene oxide after being washed by aqua DM until reached neutral $\mathrm{pH}$ and dried over heat in temperature $100^{\circ} \mathrm{C}$ by using oven for 24 hours.

\section{Cyclic Voltammetry and Characterization}

Using 8:2 ratio for mixed compound of GO and organic insulator, paraffin. This optimum composition then measured by voltammeter in measuring condition at $10 \mathrm{~s}$ deposition time, $300 \mathrm{mV} / \mathrm{s}$ scan rate and $\mathrm{pH} 9$ for sample.

Physical characterization has a primer function to know the morphological surface of its control (graphite) and GO to be tested using SEM-EDX JEOL JSM 6510 LA. Crystalline structure has been tested using XRD Phillips PANalytical in National Nuclear Power Agency (BATAN), with 2theta angle at $10^{\circ}-80^{\circ}$. While chemical characterization has been done to show functional group, using FTIR at Institute Technology of Sepuluh November Surabaya.

\section{RESULTS AND DISCUSSION}

A. Synthesized Graphite Oxide and Graphene Oxide

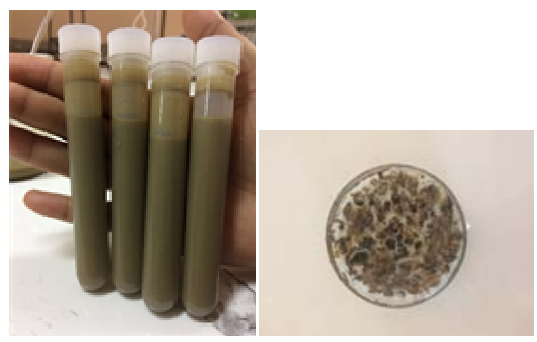

Fig. 1. The process of producing graphite oxide from graphite

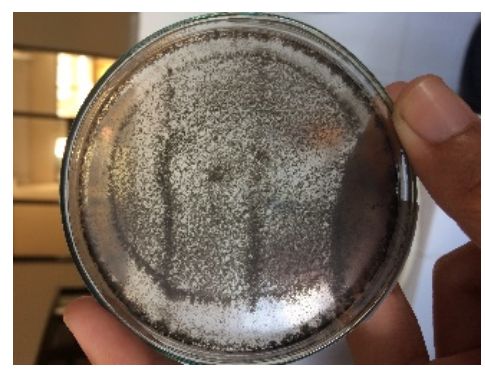

Fig. 2. Graphene Oxide that had been produced from graphite oxide

\section{B. Characterization Results}

\section{1) XRD Characterization Result}

Characterization of crystal from synthesis has been done with using XRD PANalytical instrument at National Nuclear Power Agency (BATAN), Serpong. This experiment has been tested using $\mathrm{Cu}-\mathrm{K} \alpha$ anode in wavelength $1.54 \AA$ \& on angle degree 2theta at $10^{\circ}$ until $80^{\circ}$. This obtained by comparing precursor (graphite) with GO that shown in fig. 3 and 4.

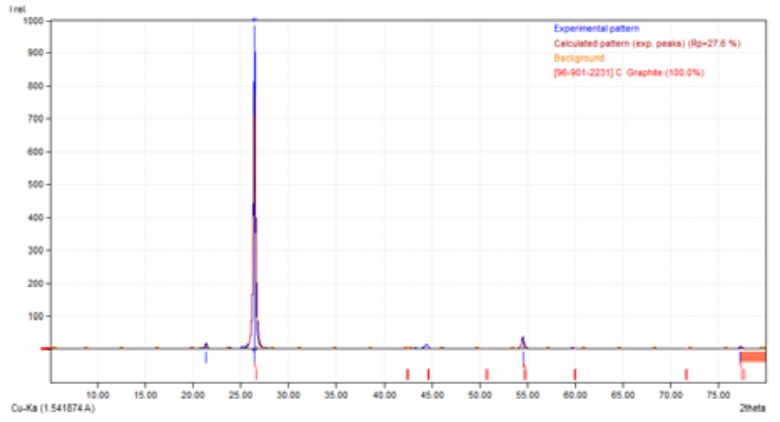

Fig. 3. Diffractogram result of 300 mesh grapihte 


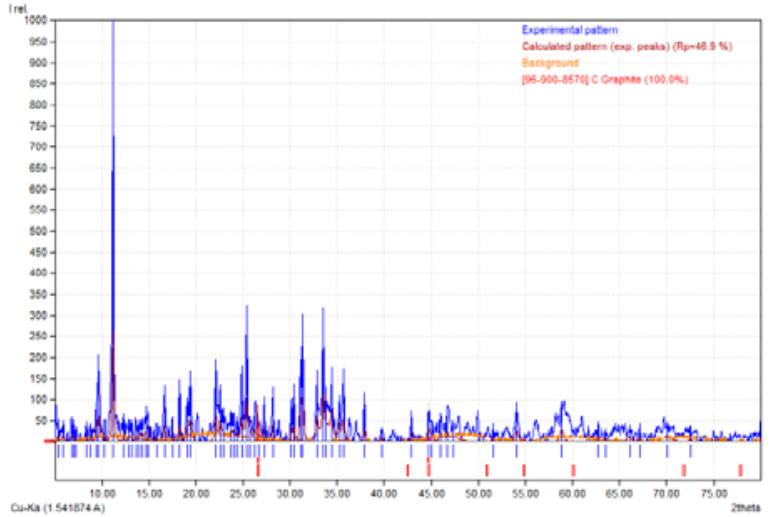

Fig. 4. Diffractogram result of synthesized GO

These results later to be counted using Debye-Scherer equation to know crystalline size that expected as a nanomaterial. Based on calculation at each angle produced by diffractogram, changes of crystalline size happened at closure angles. At the same angle, which $26.4^{\circ}$ (graphite) and $26.6^{\circ}(\mathrm{GO})$, the crystalline size changed after synthesizing treatment. Crystalline size at angle $26.4^{\circ}$ is 5.89 while in $26.6^{\circ}$ angle is 3.707. Shifting angle that happened caused by sampling preparation and sample size that produced was not actually even. This impacted for angle result, which being stroke from X-ray instrument.

\section{2) SEM Characterization Result}

Characterization for morphological structure has using SEM is to know graphite that has been changed before and after synthesizing. Magnification that used in each test is $3.500 \mathrm{X}$, respectively. The test is done at PSTBM-BATAN with instrument series SEM-EDS JEOL JSM 6510LA (Fig. 5 and 6).

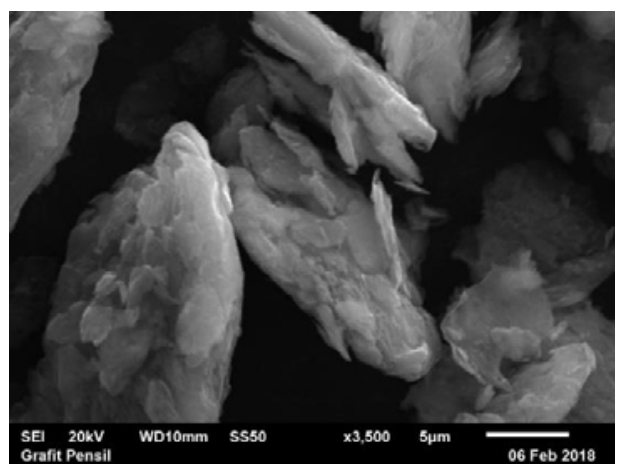

FIG. 5. SEM picture of graphite 300 mesh before synthesis.

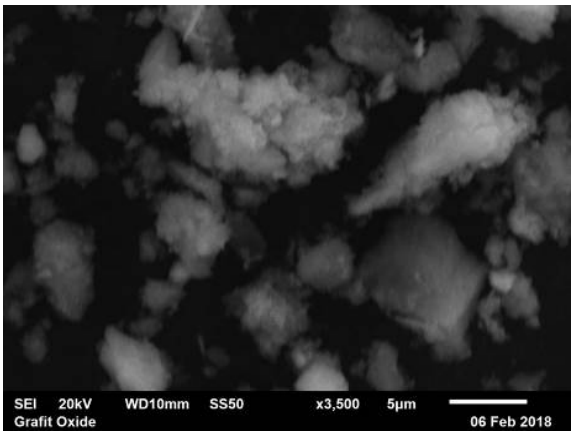

Figure 6. SEM picture of GO after synthesis

\section{3) FTIR Characterization Result}

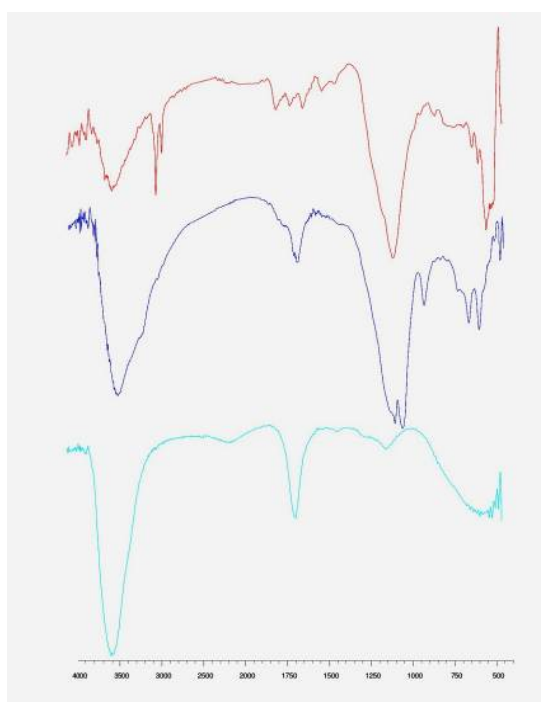

Fig. 7. Result of using FTIR on sample test of graphite, graphite oxide and GO.

Based on the result from fig. 7, there are some shifting wavenumber on each step of synthesis. First, shift occurred at peak number $100 \mathrm{~cm}^{-1}$ to $1003 \mathrm{~cm}^{-1}$ respectively for graphite and graphite oxide. Whereas on the same wavenumber cannot be shown by GO. Same wavenumber peak has shown on $1624 \mathrm{~cm}^{-1}$ until $1654 \mathrm{~cm}^{-1}$ for graphite with $1633 \mathrm{~cm}^{-1}$ represented for graphite oxide and $1635 \mathrm{~cm}^{-1}$ for GO. Nevertheless, this peak that caused shifting effect occurred at $3450 \mathrm{~cm}^{-1}$ stands for graphite, $3392 \mathrm{~cm}^{-1}$ for graphite oxide and $3446 \mathrm{~cm}^{-1}$ for GO. 
4) Voltammogram Analysis for Coefficient Diffusion of Working Electrode

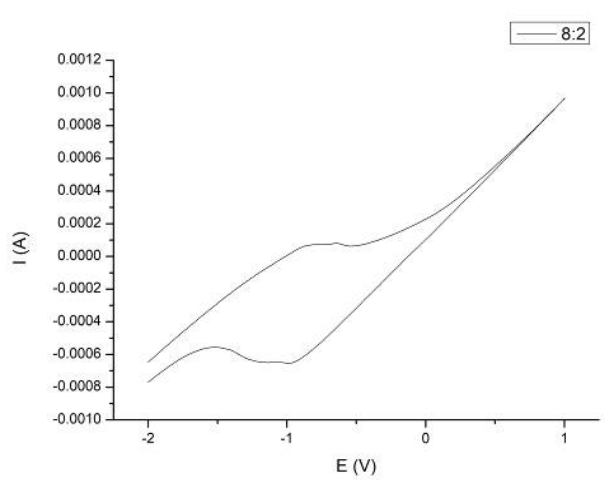

Fig. 8. Voltammogram at optimum condition

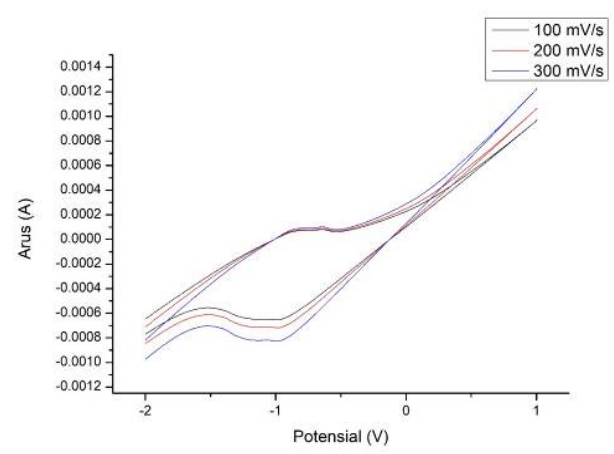

Fig. 9. Varied Scan Rate at $\mathrm{pH} 9$ at Optimum Composition

From fig. 8 and 9, optimum $\mathrm{pH}$ of alkaline water is $\mathrm{pH}$ 9. The test has been done based on optimum composition of electrode $8: 2$ in scan rate $100 \mathrm{mV} / \mathrm{s}$ at deposition time 10s. The peak result at $\mathrm{pH} 9$ tends at low level and there are no sharp peak while scanning from toward current to back current. The result of the fig. 9 has been shown on this table below (TABLE 1).

TABLE. 1. VARIED SCAN RATE AT OPTIMUM CONDITION

\begin{tabular}{|c|c|c|c|c|}
\hline Scan Rate & Ipc (A) & Epc (V) & Ipa (A) & Epa (V) \\
\hline $100 \mathrm{mV} / \mathrm{s}$ & $-6.50 \times 10^{-4}$ & -0.964 & $1.948 \times 10^{-6}$ & -1.0062 \\
\hline $200 \mathrm{mV} / \mathrm{s}$ & $-7.18 \times 10^{-4}$ & -0.988 & $2.143 \times 10^{-6}$ & -1.0062 \\
\hline $300 \mathrm{mV} / \mathrm{s}$ & $-8.26 \times 10^{-4}$ & -0.994 & $6.468 \times 10^{-6}$ & -1.0002 \\
\hline
\end{tabular}

By the following data, we can calculate coefficient diffusion for the highest value of varied scan rate using the Randles-Sevcik equation.

$f p=2.686 \times 10^{2} n^{8 / 2} \cdot A c^{0} D^{1 / 2} v^{1 / 2}$

From this equation, the results for calculated coefficient diffusion of each varied scan rate is showed by the table 2 below.
TABLE 2. COEFFICIENT DIFFUSION OF VARIED SCAN RATE

\begin{tabular}{|c|c|c|}
\hline Scan Rate $(\mathbf{m V} / \mathbf{s})$ & $\mathrm{D} \mathrm{i}_{\mathrm{pc}}\left(\mathrm{cm}^{2} / \mathbf{s}\right)$ & $\mathrm{D}_{\mathrm{pa}}\left(\mathrm{cm}^{2} / \mathbf{s}\right)$ \\
\hline 100 & 27,19 & $\mathbf{1 , 4 8 7}$ \\
\hline 200 & 12,75 & $\mathbf{0 , 6 9 5}$ \\
\hline 300 & $\mathbf{5 , 5 0}$ & $\mathbf{0 , 4 8 7}$ \\
\hline
\end{tabular}

Compare with carbon paste electrode, GO paste electrode can be proven to be better for conductivity based on voltammogram result. The following result is the comparison between carbon paste electrode with GO paste, shown by figure 10 , followed by voltammogram data shown by table 3

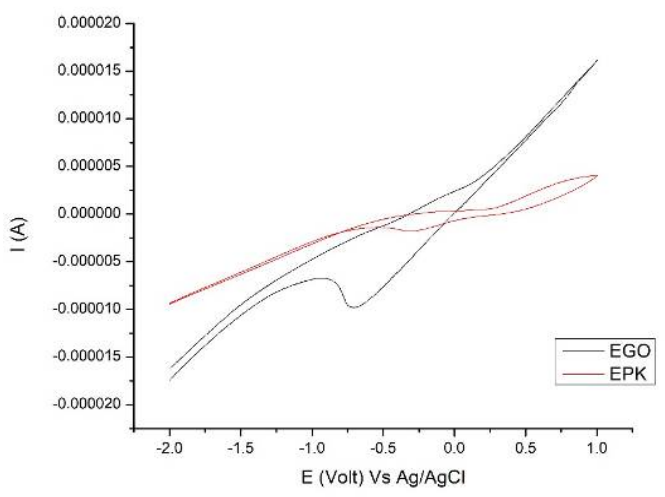

Fig. 10. Comparison between carbon paste electrode with GO electrode

TABLE 3. VOLTAMMOGRAM FOR COMPARISON ELECTRODE

\begin{tabular}{|c|c|c|c|c|}
\hline Sample & Ipc (A) & Epc (V) & Ipa (A) & Epa (V) \\
\hline Carbon & $2.63 \times 10^{-}$ & -0.10757 & $-1.7823 \times$ & -0.29205 \\
Paste & 7 & & $10^{-6}$ & \\
\hline GO Paste & $2.01 \times 10^{-}$ & -0.059967 & $-9.762 \times$ & -0.72647 \\
& 6 & & $10^{-6}$ & \\
\hline
\end{tabular}

\section{CONCLUSION}

From the result of this research, it proved that graphene oxide can be used as a working electrode that has better performance in conductivity, represented by coefficient diffusion that enhance activity of electron transfer.

\section{ACKNOWLEDGMENT}

Thank you to Prof. Dr. Suyatno, M.Si as chairman of the Chemistry Department of Universitas Negeri Surabaya and Prof. Dr. Suyono., M.Pd as dean of FMIPA Universitas Negeri Surabaya.

\section{REFERENCES}

[1] Ambrosi A, Chua CK, Bonanni A, et al. Electrochemistry of graphene and related materials. Chemical Reviews. 2014;114(14):7150-88 
[2] Geim, A.K. and Novoselov, K.S. The Rise of Graphene. Nature Materials, 2007, 6, 183-191. http://dx.doi.org/10.1038/nmat1849

[3] Gethers, M.L., Thomas, J.C., Jiang, S., Weiss, N.O., Duan, X., Goddardlll, W.A. and Weiss, P.S. Holey Graphene as a Weed Barrier for Molecules. ACS Nano, 2015, 9, 1090910915. http://dx.doi.org/10.1021/acsnano.5b03936

[4] Eko Y, Pirim S. Producing Modified Carbon Paste Electrode for $\mathrm{Cr}$ (VI) Analyzing with Cyclic Stripping Voltammetry. State University of Surabaya: Indonesia, 2014.
[5] Ardi dan Setiarso R. Use zeolite as modifier carbon electrode pasta for analysis Cd (II). In: Cyclic Stripping Voltammetry. State University of Surabaya: Indonesia, 2016;5(3)

[6] S. P. Kounaves. Handbook of Intrumental Techniques for Analytical Chemistry. Department Chemistry. Tufts University. Chapt. 37. Pp 709-725. 